\title{
Impactos da pandemia do COVID-19 em crianças com Transtorno do Espectro do
}

\section{Autismo: Uma revisão integrativa}

Impacts of the COVID-19 pandemic on children with Autism Spectrum Disorder: An integrative review

Impactos de la pandemia COVID-19 en niños con Transtorno del Espectro Autista: Una revisión integrativa

Milena Cordeiro de Freitas ORCID: https://orcid.org/0000-0003-0208-9400 Faculdade Cearense, Brasil

E-mail: barra.milenafreitas@hotmail.com

Thayane Cintra Lemos

ORCID: https://orcid.org/0000-0002-8918-7531 Universidade Federal do Espirito Santo, Brasil E-mail: thayane309@gmail.com

Vitória Luiza Cavalcanti de Lima ORCID: https://orcid.org/0000-0002-1311-2832 Faculdade Cearense, Brasil

E-mail: vit_oria_cavalcanti@hotmail.com

Paula Ermans de Oliveira

ORCID: https://orcid.org/0000-0002-2735-5202 Universidade Portiguar, Brasil

E-mail: paulaaermans@gmail.com

Kamila de Castro Morais

ORCID: https://orcid.org/0000-0002-3564-7993

Universidade Regional do Cariri, Brasil

E-mail: kamilacastromorais@gmail.com

Antônio Diego Costa Bezerra

ORCID: https://orcid.org/0000-0002-2441-2961

Centro Universitário Unifanor, Brasil Fundação Oswaldo Cruz, Brasil

E-mail: diegocostamjc@gmail.com

Eduardo Odonete Marques

ORCID: https://orcid.org/0000-0002-4739-6474

Universidade Federal do Piauí, Brasil

E-mail: eduardomarques@ufpi.edu.br

Sandryelle de Andrade Rodrigues ORCID: https://orcid.org/0000-0002-0878-3885 Centro Universitário Doutor Leão Sampaio, Brasil

E-mail: sandryellerodrigues24@gmail.com

André Sousa Rocha

ORCID: https://orcid.org/0000-0002-0185-9699

Universidade São Francisco, Brasil

E-mail: andresousarocha9@gmail.com

Cidianna Emanuelly Melo do Nascimento

ORCID: https://orcid.org/0000-0001-5477-4413

Universidade Estadual do Ceará, Brasil

E-mail: profa.cidianna.melo@gmail.com

\section{Resumo}

A pandemia do COVID-19 teve a eclosão de casos confirmados em dezembro de 2019. Ademais, de acordo com a Organização Mundial da Saúde (OMS), o vírus SARS-CoV-2 foi declarado como Emergência de Saúde Pública de Importância Internacional. É necessário compreender os impactos causados nas questões das relações dos diferentes grupos sociais. O Transtorno do Espectro do Autismo (TEA) é um transtorno do neurodesenvolvimento caracterizado por déficits que permeiam a comunicação e interação social. Logo, para esse grupo específico, viver à pandemia pode acarretar diversos problemas. O presente estudo teve como objetivo evidenciar informações acerca dos impactos da 
Research, Society and Development, v. 10, n. 3, e57010313664, 2021

(CC BY 4.0) | ISSN 2525-3409 | DOI: http://dx.doi.org/10.33448/rsd-v10i3.13664

pandemia do COVID-19 em crianças com TEA. Trata-se de uma revisão integrativa da literatura (RIL). A busca de artigos ocorreu em fevereiro de 2021 na base de dados PUBMED e na biblioteca BVS com descritores: Child, COVID-19, Autism Spectrum Disorder. Foram recuperados 89 artigos e apenas 12 permaneceram para leitura na íntegra. A análise dos estudos selecionados evidenciou impactos positivos e negativos em crianças com TEA durante a pandemia. Todo esse contexto contribuiu principalmente para o desenvolvimento de problemas de comportamento e aumento de ansiedade e estresse. Nesse sentido, é importante que a família implemente uma nova rotina e trate calmamente da situação.

Palavras-chave: Criança; COVID-19; Transtorno do espectro autista.

\begin{abstract}
The COVID-19 pandemic had confirmed outbreaks in December 2019. Furthermore, according to the World Health Organization (WHO), the SARS-CoV-2 virus was declared a Public Health Emergency of International Importance. It is necessary to understand the impacts caused on the issues of the relationships of different social groups. Autism Spectrum Disorder (ASD) is a neurodevelopmental disorder characterized by deficits that permeate communication and social interaction. Therefore, for this specific group, living with the pandemic can cause several problems. The present study aimed to highlight information about the impacts of the COVID-19 pandemic on children with ASD. This is an integrative literature review (RIL). The search for articles occurred in February 2021 in the PUBMED database and in the VHL library with descriptors: Child, COVID-19, Autism Spectrum Disorder. 89 articles were recovered and only 12 remained for full reading. The analysis of the selected studies showed positive and negative impacts on children with ASD during the pandemic. This whole context contributed mainly to the development of behavioral problems and increased anxiety and stress. In this sense, it is important for the family to implement a new routine and calmly deal with the situation.
\end{abstract}

Keywords: Kid; COVID-19; Autistic spectrum disorder.

\title{
Resumen
}

La pandemia COVID-19 había confirmado brotes en diciembre de 2019. Además, según la Organización Mundial de la Salud (OMS), el virus SARS-CoV-2 fue declarado Emergencia de Salud Pública de Importancia Internacional. Es necesario comprender los impactos causados en los temas de las relaciones de diferentes grupos sociales. El trastorno del espectro autista (TEA) es un trastorno del desarrollo neurológico caracterizado por déficits que impregnan la comunicación y la interacción social. Por lo tanto, para este grupo específico, vivir con la pandemia puede causar varios problemas. El presente estudio tuvo como objetivo resaltar información sobre los impactos de la pandemia COVID-19 en niños con TEA. Ésta es una revisión integradora de la literatura (RIL). La búsqueda de artículos se produjo en febrero de 2021 en la base de datos PUBMED y en la biblioteca de la BVS con descriptores: Niño, COVID-19, Trastorno del espectro autista. Se recuperaron 89 artículos y solo quedaron 12 para lectura completa. El análisis de los estudios seleccionados mostró impactos positivos y negativos en los niños con TEA durante la pandemia. Todo este contexto contribuyó principalmente al desarrollo de problemas de conducta y al aumento de la ansiedad y el estrés. En este sentido, es importante que la familia implemente una nueva rutina y maneje con calma la situación.

Palabras clave: Niño; COVID-19; Trastorno del espectro autista.

\section{Introdução}

A pandemia do COVID-19 teve a eclosão de casos confirmados em dezembro de 2019, em Wuhan, uma província chinesa. Diante disso, de acordo com a etimologia do termo pandemia, fica designado por ser epidemias que espalham facilmente por diversos países, logo sendo uma epidemia global. Ademais, de acordo com a Organização Mundial da Saúde (OMS), o vírus SARS-CoV-2 (Síndrome Respiratória Aguda Grave Coronavírus 2) foi declarado como Emergência de Saúde Pública de Importância Internacional (Oms, 2020).

É notório que a principal forma de prevenção e cuidados da doença têm sido através de atitudes de higiene, como o ato de higienizar as mãos com frequência e da forma correta, o uso de máscaras e o isolamento social (Opas, 2020).

Devido os estudos relacionados ao COVID-19 serem recentes, ainda há indagações quanto à sua relação e impactos em casos de crianças com Transtorno do Espectro do Autismo (TEA). Entretanto, é necessário compreender os impactos causados nas questões das relações sociais dos diferentes grupos sociais (Farias \& Leite Júnior, 2020). 
Research, Society and Development, v. 10, n. 3, e57010313664, 2021

(CC BY 4.0) | ISSN 2525-3409 | DOI: http://dx.doi.org/10.33448/rsd-v10i3.13664

O TEA é um transtorno do neurodesenvolvimento caracterizado por déficits que permeiam a comunicação e interação social. Por esse viés, pensar que o TEA é degenerativo torna-se um pensamento equivocado, visto que a aprendizagem continua ao longo da vida (Apa, 2013).

O TEA é comumente identificado na infância e possui como principais características a dificuldade de interação social e comunicação, além de dificuldades comportamentais (Apa, 2013). Logo, para esse grupo específico, viver à pandemia e as suas ramificações e impactos podem acarretar diversos problemas, principalmente quando trata-se de crianças (Houting, 2020).

Por esse viés, crianças diagnosticadas com TEA tornam-se vulneráveis ao COVID-19, considerando todos os aspectos de interações sociais que podem agravar o quadro clínico. Além disso, segundo a Sociedade Brasileira de Pediatria (SBP) os impactos da pandemia do COVID-19 nas rotinas familiares fazem ser necessário reestruturar a rotina e as atividades comportamentais. Entretanto, a abrupta mudança de rotina pode acarretar às crianças sensibilizadas (Brito, Almeida, Crenze, Alves, Lima \& Abranches, 2020).

Ademais, devido à alteração de rotina em decorrência da pandemia, as famílias de portadores de TEA precisam explorar a paciência e criatividade tanto das crianças quanto dos cuidadores, considerando que a repetitividade e monotonia é presente nas brincadeiras, o que torna o interesse das crianças com TEA em padrões limitados (Barbosa, 2020).

Diante disso, também é necessário avaliar quanto às práticas educacionais, considerando que o principal impacto da pandemia é o distanciamento social, logo os grupos terapêuticos e aulas escolares foram suspensas, e com essa alteração de rotina, o ideal é que os responsáveis busquem manter os horários de estudos e continuem o conteúdo programático de acordo com as atividades propostas, a fim de diminuir ainda mais os impactos em crianças com TEA (Brito et al., 2020).

Sendo assim, diante do exposto, é relevante discutir acerca dos impactos da pandemia do COVID-19 em crianças com TEA, tendo em vista que as suas rotinas ficaram totalmente comprometidas devido ao isolamento social e a gravidade da pandemia. Portanto, o presente estudo teve como objetivo evidenciar na literatura científica informações acerca dos impactos da pandemia do COVID-19 em crianças com TEA.

\section{Metodologia}

O estudo trata-se de uma revisão integrativa de literatura (RIL) com abordagem metodológica qualitativa (Martinelli, 2019). Esse tipo de estudo é um método que proporciona a síntese dos conhecimentos adquiridos, assim como também faz um levantamento bibliográfico (Souza, Silva \& Carvalho, 2010). Logo, para a elaboração do presente estudo, fez-se necessário seguir as seguintes etapas: definição do objeto de estudo e elaboração da pergunta norteadora; definição dos critérios de inclusão e exclusão e a busca na literatura; coleta de dados e organização das informações encontradas; reflexão e avaliação crítica das informações coletadas; discussão dos resultados e apresentação da RIL (Mendes, Silveira \& Galvão, 2008).

O ponto de partida deu-se por meio da utilização da estratégia PICo (acrônimo de população, interesse e contexto). A sintaxe de aplicação desta estratégia está descrita no Quadro 1, no qual traz a descrição dos acrônimos correspondentes ao estudo, bem como os Descritores em Ciências da Saúde (DeCS) e Medical Subject Headins (MeSH) a serem utilizados posteriormente nas estratégias de busca. 
Research, Society and Development, v. 10, n. 3, e57010313664, 2021

(CC BY 4.0) | ISSN 2525-3409 | DOI: http://dx.doi.org/10.33448/rsd-v10i3.13664

Quadro 1 - Estratégia PICo.

\begin{tabular}{|c|c|c|c|c|}
\hline Etapa & Definição & Descrição & $\begin{array}{c}\text { Descritores em Ciências da } \\
\text { Saúde (DeCS) }\end{array}$ & $\begin{array}{c}\text { Medical Subject Headings } \\
\text { (MeSH) }\end{array}$ \\
\hline $\mathbf{P}$ & População & Crianças & Criança & Child \\
\hline I & Interesse & $\begin{array}{c}\text { Transtorno do Espectro do } \\
\text { Autismo }\end{array}$ & Transtorno do Espectro Autista & Autism Spectrum Disorder \\
\hline Co & Contexto & Pandemia do COVID-19 & COVID-19 & COVID-19 \\
\hline
\end{tabular}

Fonte: Autores (2021).

A partir da consideração da estratégia PICo, o primeiro elemento (P) denomina-se para crianças; o segundo (I) para com Transtorno do Espectro do Autismo e o terceiro (Co) volta-se para a pandemia do COVID-19. Dessa forma, a pergunta norteadora do estudo partiu da seguinte questão: Quais são os impactos da pandemia da COVID-19 em crianças com Transtorno do Espectro do Autismo?

A busca de estudos ocorreu em fevereiro de 2021 na base de dados U.S. National Library of Medicine and the National Institutes Health (PUBMED) e na Biblioteca Virtual em Saúde (BVS). Quanto aos descritores, foram selecionados na Medical Subject Headings (MeSH): Child, COVID-19, Autism Spectrum Disorder. Foi aplicado o operador booleano AND (Quadro 2).

Quadro 2 - Aplicação da estratégia de busca.

\begin{tabular}{|c|c|c|c|c|}
\hline Data & Base de Dados & Estratégia & Termos de Busca & Resultados \\
\hline $22 / 02 / 2021$ & PubMed & PICo & $\begin{array}{c}\text { (Child*) AND (COVID-19)) AND } \\
\text { (Autism Spectrum Disorder) }\end{array}$ & 55 \\
\hline $23 / 02 / 2021$ & BVS & PICo & $\begin{array}{c}\text { Child* AND “COVID-19” AND } \\
\text { "Autism Spectrum Disorder” }\end{array}$ & 34 \\
\hline
\end{tabular}

Fonte: Autores (2021).

Os critérios de inclusão voltaram-se para artigos gratuitos disponíveis na íntegra e redigidos em português, espanhol ou inglês. Os critérios de exclusão foram definidos em estudos de revisão; relatos de caso; cartas ao editor; cartas; editoriais; comentários; casos clínicos; estudos que tratam sobre os pais/cuidadores de crianças com TEA; uso da telemedicina; uso de tecnologias; recomendações sobre a pandemia para autistas; não citar crianças com TEA; diagnóstico de autismo na gravidez; estudos que abordaram outro tipo de transtorno; capítulos de livros e outras publicações que não passaram por revisão dos pares.

Foram identificados 89 estudos, sendo 34 na BVS e 55 na PubMed. Na fase de identificação foram excluídos 26 textos por duplicidade e 3 por não seguirem os critérios de inclusão ( 2 artigos pagos e 1 na língua francesa). Assim, na triagem foram avaliados 60 estudos. Destes, após leitura dos títulos e resumos, 10 foram excluídos por tratar sobre os pais/cuidadores de crianças com TEA; oito eram sobre telemedicina; dois sobre tecnologias; sete eram revisões; um relato de caso; três eram recomendações sobre a COVID-19; um não citava crianças com TEA; dois eram editoriais; um tratava do diagnóstico de TEA em gestantes; três eram comentários; um caso clínico; uma carta; duas cartas ao editor; um protocolo; quatro sobre atuação dos 
Research, Society and Development, v. 10, n. 3, e57010313664, 2021

(CC BY 4.0) | ISSN 2525-3409 | DOI: http://dx.doi.org/10.33448/rsd-v10i3.13664

profissionais da saúde e um estudo não passou pela revisão de pares. Portanto, 12 artigos foram incluídos na fase de elegibilidade e todos compuseram a amostra final para a elaboração desta RIL.

Para extração e análise dos dados foi elaborado um quadro no Microsoft Excel que continha: autores, local/ano, título, objetivo, tipo de estudo/nível de evidência e impactos do COVID-19 em crianças com TEA (Quadro 4).

Para sistematizar a busca e seleção dos artigos coletados fez-se necessário utilizar a recomendação de Preferred Reporting Items for Systematic Reviews and MetaAnalyses (PRISMA) (Moher, Liberati, Tetzlaff \& Altman, 2009), conforme segue abaixo (Quadro 3):

Quadro 3 - Fluxograma (PRISMA)

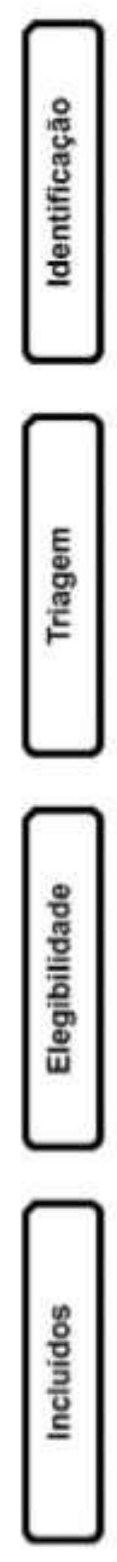

29 Artigos excluidos ( 26 por duplicidade, 2 por serem pagos e I estar na lingua francesa) dados $(\mathbf{n}=89)$

PubMed: 55

BVS: 34
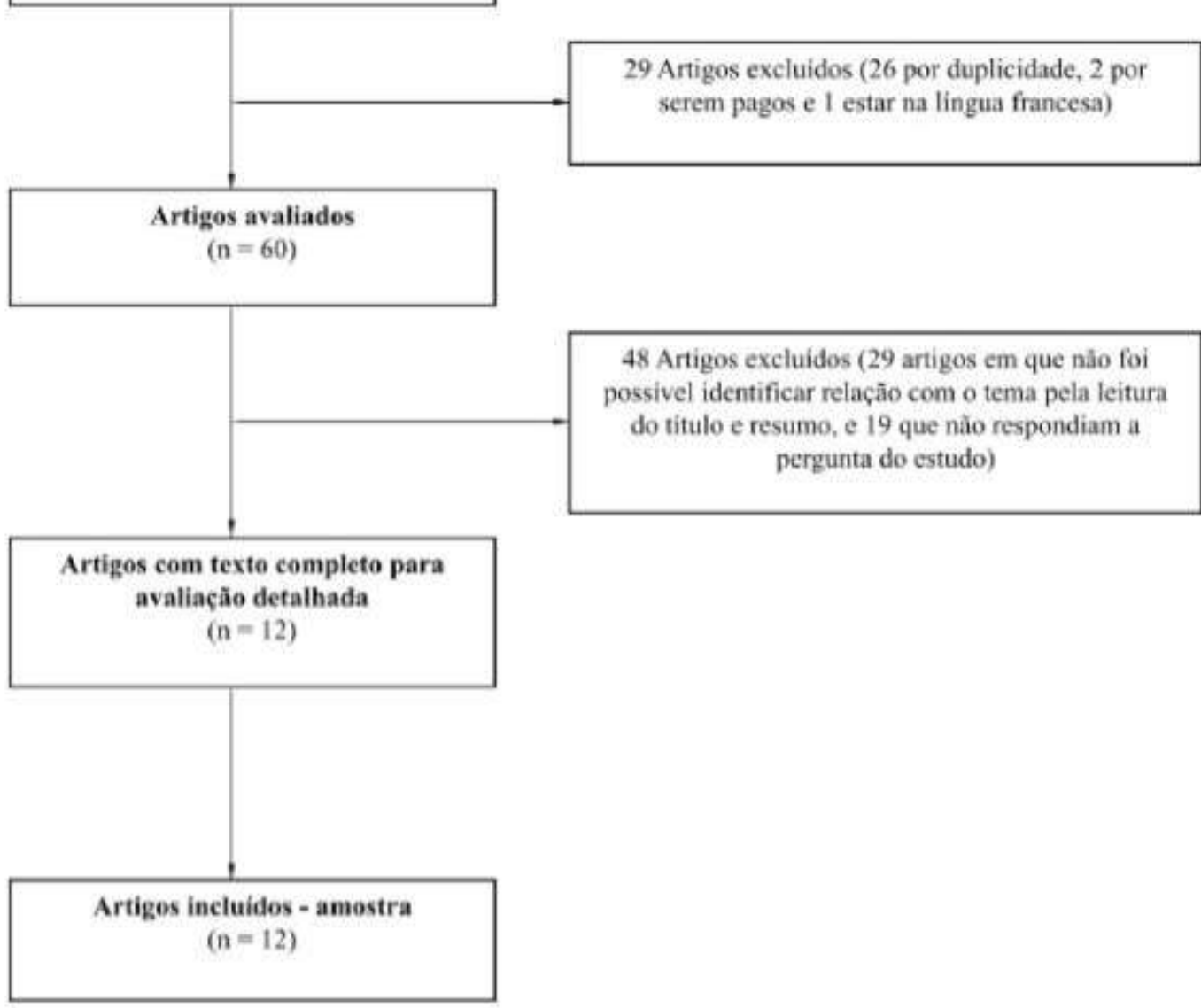

Fonte: Autores (2021). 


\section{Resultados e Discussão}

Foram recuperados 89 artigos e apenas 12 permaneceram para leitura na íntegra. Dos 12 artigos analisados, três foram desenvolvidos na Itália, dois nos Estados Unidos, dois na Turquia, um em Portugal, um no Japão, um na Espanha, um no Reino Unido e um na Irlanda. Destes, 11 foram publicados em inglês $(91,6 \%)$ e somente um em espanhol. A análise dos estudos selecionados evidenciou impactos positivos e negativos em crianças com Transtorno do Espectro do Autismo (TEA) durante a pandemia do COVID-19, sendo os positivos: desenvolvimento de autonomia para cuidarem de si (vestir-se, comer, higiene pessoal); participação na rotina da casa; algumas crianças continuaram a ter ganhos de desenvolvimento, principalmente na linguagem; o comportamento de "compartilhar e buscar o outro" aumentou e melhorou. Entretanto, os impactos negativos incluem: problemas de comportamento, causados por ansiedade, irritabilidade, obsessão, hostilidade e impulsividade; aumento dos níveis de ansiedade e estresse devido, sobretudo, a mudanças na rotina; piora na gestão da emoção; perda pelo menos um serviço de terapia ABA ou educação especial; aumento no tempo de uso de mídias digitais e internet; problemas para entender o que é COVID-19 e as medidas necessárias de distanciamento social e higiene; aumento do retraimento social e alterações do sono.

A Organização Mundial da Saúde (OMS) declarou no dia 11 de março de 2020 a pandemia do novo coronavírus (Oms, 2020) e com isso, as medidas de isolamento social tiveram início a fim de conter a propagação do vírus e diminuir o número de casos positivos. As medidas de distanciamento social afastaram as crianças da terapia ABA e da escola, somente algumas receberam acesso por meio da teleducação (Colizzi, Sironi, Antonini, Ciceri, Bovo \& Zoccante, 2020). Todo esse contexto alterou as rotinas das crianças com TEA e essas alterações são desafios significativos para esses indivíduos (Baumer \& Spence, 2018), o que contribuiu para o desenvolvimento de problemas de comportamento, tais como: aumento da retração social, ansiedade, estresse, irritabilidade, hipersensibilidade, alterações de sono e apetite, entre outros (Kentaro, Rie, Kiwamu, Ayumi, Fumie \& Shu-Ichi, 2020; Tuba, Ceymi \& Herdem, 2020; O’Sullivan, 2021). Houve também o uso excessivo da Internet entre crianças com TEA (Kentaro, 2020), sendo que o consumo massivo de conteúdos sobre a situação da epidemia (número de casos, número de mortos) pode gerar ansiedade, pânico e levar à depressão, e pode gerar uma forma de vício, um transtorno que gera dependência (Deslandes \& Coutinho, 2020).

Entretanto, com o advento da pandemia do novo Coronavírus e a necessidade de manter o isolamento, as crianças passaram a participar com mais frequência da nova rotina familiar, como por a mesa ou escolher uma atividade para fazer, e a se comunicarem mais com os pais (Mumbardó-Adam, Barnet-López \& Balboni, 2021). Poucas crianças desenvolveram novas estereotipias como puxar as próprias orelhas ou falar com um tom de voz mais alto (Mumbardó-Adam et al., 2021), e algumas experimentaram melhorias na comunicação, socialização e autonomia pessoal durante momentos de lockdown (Sergi, Mingione, Ricci, Cavallaro, Russo, Corrivetti, Operto \& Frolli, 2021).

A análise dos estudos (Quadro 4) indica que a alteração na rotina da criança com TEA é o principal fator para alterações problemáticas de comportamento que acarretam em ansiedade e estresse para a criança. Muitas tiveram sua educação especial e o tratamento ABA interrompidos, o que prejudicou seu desenvolvimento ou o estagnou. A maioria das crianças conseguiu ter maior contato com os pais e maior autonomia. Sendo assim, foi encontrada a necessidade de estudos a respeito de ferramentas lúdicas ou tecnológicas que auxiliem os pais/cuidadores de crianças com TEA a minimizarem os impactos negativos do isolamento social e estudos sobre a adoção de teleducação e telessaúde para esse público. 
Quadro 4 - Dados dos estudos selecionados para a RIL.

\begin{tabular}{|c|c|c|c|c|c|}
\hline Autores & Local/Ano & Título & Objetivo & $\begin{array}{l}\text { Tipo de } \\
\text { Estudo/Nível } \\
\text { de Evidência }\end{array}$ & $\begin{array}{l}\text { Impactos da pandemia do COVID- } \\
19 \text { em crianças autistas }\end{array}$ \\
\hline Amorim et al. & $\begin{array}{l}\text { Portugal, } \\
2020\end{array}$ & $\begin{array}{l}\text { Impacto de la COVID- } \\
19 \text { en niños con } \\
\text { trastorno del espectro } \\
\text { autista }\end{array}$ & $\begin{array}{l}\text { Explorar como as crianças } \\
\text { com TEA e seus pais } \\
\text { vivenciaram o isolamento } \\
\text { social durante o período do } \\
\text { surto de COVID- } 19 .\end{array}$ & $\begin{array}{l}\text { Estudo } \\
\text { observacional, } \\
\text { transversal e } \\
\text { analítico. }\end{array}$ & $\begin{array}{l}\text { Crianças com TEA tiveram } \\
\text { predominantemente mudanças de } \\
\text { comportamento; um impacto negativo } \\
\text { na gestão da emoção; apresentaram } \\
\text { níveis mais elevados de ansiedade. As } \\
\text { crianças com TEA que não } \\
\text { mantiveram a rotina apresentaram } \\
\text { níveis médios de ansiedade mais } \\
\text { elevados do que as crianças que } \\
\text { mantiveram a rotina. As causas da } \\
\text { mudança de comportamento foram } \\
\text { ansiedade, irritabilidade, obsessão, } \\
\text { hostilidade e impulsividade. }\end{array}$ \\
\hline Colizzi et al. & Itália, 2020 & $\begin{array}{l}\text { Psychosocial and } \\
\text { Behavioral Impact of } \\
\text { COVID-19 in } \\
\text { Autism Spectrum } \\
\text { Disorder: An Online } \\
\text { Parent Survey }\end{array}$ & $\begin{array}{l}\text { Investigar o impacto da } \\
\text { pandemia COVID-19 em } \\
\text { indivíduos com TEA, se } \\
\text { quaisquer características } \\
\text { sociodemográficas ou clínicas } \\
\text { pré-pandêmicas poderiam } \\
\text { prever um resultado negativo } \\
\text { e caracterizar narrativamente } \\
\text { suas necessidades. }\end{array}$ & $\begin{array}{l}\text { Estudo } \\
\text { quantitativo }\end{array}$ & $\begin{array}{l}\text { Apresentam problemas de } \\
\text { comportamento mais intensos e } \\
\text { frequentes. }\end{array}$ \\
\hline Jeste et al. & $\begin{array}{l}\text { Estados } \\
\text { Unidos, } \\
2020\end{array}$ & $\begin{array}{l}\text { Changes in access to } \\
\text { educational and } \\
\text { healthcare services for } \\
\text { individuals with } \\
\text { intellectual and } \\
\text { developmental } \\
\text { disabilities during } \\
\text { COVID19 restrictions }\end{array}$ & $\begin{array}{l}\text { Capturar mudanças no acesso } \\
\text { a serviços de saúde e serviços } \\
\text { educacionais para indivíduos } \\
\text { com deficiência intelectual e } \\
\text { de desenvolvimento que } \\
\text { ocorreram logo após o início } \\
\text { das restrições e para pesquisar } \\
\text { famílias sobre recursos que } \\
\text { poderiam melhorar os } \\
\text { serviços para esses } \\
\text { indivíduos. }\end{array}$ & $\begin{array}{l}\text { Estudo } \\
\text { quantitativo }\end{array}$ & $\begin{array}{l}\text { Perderam acesso a pelo menos um } \\
\text { serviço de terapia ou educação e } \\
\text { somente algumas receberam pelo } \\
\text { menos alguns serviços continuados } \\
\text { por meio de teleducação. }\end{array}$ \\
\hline Kawabe et al. & Japão, 2020 & $\begin{array}{l}\text { Excessive and } \\
\text { Problematic Internet } \\
\text { Use During the } \\
\text { Coronavirus Disease } \\
\text { 2019 School Closure: } \\
\text { Comparison Between } \\
\text { Japanese Youth With } \\
\text { and Without Autism } \\
\text { Spectrum Disorder }\end{array}$ & $\begin{array}{l}\text { Explorar a diferença no uso } \\
\text { de internet e mídia digital } \\
\text { entre crianças e adolescentes } \\
\text { com e sem TEA e comparar a } \\
\text { mudança no tempo de uso } \\
\text { nesses grupos antes e durante } \\
\text { a pandemia de COVID- } 19 .\end{array}$ & $\begin{array}{l}\text { Estudo } \\
\text { transversal e } \\
\text { caso-controle } \\
\text { pareado }\end{array}$ & $\begin{array}{l}\text { A duração do uso da mídia digital foi } \\
\text { significativamente maior no grupo } \\
\text { com TEA. Uso excessivo da Internet } \\
\text { entre crianças com TEA, } \\
\text { especialmente durante a pandemia do } \\
\text { COVID-19. A maioria das crianças } \\
\text { sofreu estresse devido à pandemia. }\end{array}$ \\
\hline $\begin{array}{l}\text { Mumbardó- } \\
\text { Adam, } \\
\text { Barnet- } \\
\text { López, } \\
\text { Balboni. }\end{array}$ & $\begin{array}{l}\text { Espanha, } \\
2021\end{array}$ & $\begin{array}{l}\text { How have youth with } \\
\text { Autism Spectrum } \\
\text { Disorder managed } \\
\text { quarantine derived } \\
\text { from COVID-19 } \\
\text { pandemic? An } \\
\text { approach to families } \\
\text { perspectives }\end{array}$ & $\begin{array}{l}\text { Se aprofundar em crianças e } \\
\text { adolescentes com TEA e } \\
\text { gestão de suas famílias no } \\
\text { período de quarentena, para } \\
\text { entender melhor suas } \\
\text { necessidades. }\end{array}$ & $\begin{array}{l}\text { Estudo } \\
\text { qualitativo }\end{array}$ & $\begin{array}{l}\text { Os jovens participavam com mais } \\
\text { frequência das rotinas das famílias, } \\
\text { como pôr a mesa ou decidir } \\
\text { atividades. Poucas crianças } \\
\text { desenvolveram novas estereotipias } \\
\text { como puxar as próprias orelhas ou } \\
\text { falar com um tom de voz mais alto, } \\
\text { mas outras apresentaram níveis mais } \\
\text { elevados de autonomia para cuidarem } \\
\text { de si (higiene pessoal, vestir-se e } \\
\text { comer) e foram mais comunicativas } \\
\text { com os pais. Sentiram falta de ir } \\
\text { passear ou ao parque, ver os parentes }\end{array}$ \\
\hline
\end{tabular}


Research, Society and Development, v. 10, n. 3, e57010313664, 2021

(CC BY 4.0) | ISSN 2525-3409 | DOI: http://dx.doi.org/10.33448/rsd-v10i3.13664

\begin{tabular}{|c|c|c|c|c|c|}
\hline & & & & & $\begin{array}{l}\text { (avós, primos), frequentar atividades } \\
\text { extracurriculares como aulas de } \\
\text { dança, brincar com os amigos e fazer } \\
\text { viagens ou caminhadas. }\end{array}$ \\
\hline $\begin{array}{l}\text { Mutluer, } \\
\text { Doenyas, } \\
\text { Genc. }\end{array}$ & $\begin{array}{l}\text { Turquia, } \\
2020\end{array}$ & $\begin{array}{l}\text { Behavioral } \\
\text { Implications of the } \\
\text { COVID-19 Process for } \\
\text { Autism Spectrum } \\
\text { Disorder, and } \\
\text { Individuals' } \\
\text { Comprehension of and } \\
\text { Reactions to the } \\
\text { Pandemic Conditions }\end{array}$ & $\begin{array}{l}\text { Investigação de como os } \\
\text { indivíduos com TEA } \\
\text { responderam ao COVID-19 } \\
\text { em termos de compreensão e } \\
\text { adesão às medidas } \\
\text { implementadas; mudanças em } \\
\text { seus problemas } \\
\text { comportamentais; e como os } \\
\text { níveis de ansiedade de seus } \\
\text { cuidadores se relacionam com } \\
\text { essas mudanças } \\
\text { comportamentais. }\end{array}$ & $\begin{array}{l}\text { Estudo } \\
\text { quantitativo }\end{array}$ & $\begin{array}{l}\text { Problemas para entender o que é } \\
\text { COVID-19 e as medidas necessárias. } \\
\text { Enfrentaram desafios na } \\
\text { implementação de regulamentações } \\
\text { relacionadas ao distanciamento social } \\
\text { e higiene na pandemia. A maioria } \\
\text { deixou de receber educação especial } \\
\text { neste período. Apresentação clínica } \\
\text { relacionada à Covid-19 que se } \\
\text { assemelha ao PTSD em indivíduos } \\
\text { com TEA em termos de aumento de } \\
\text { estereotipias, agressão, } \\
\text { hipersensibilidade, problemas } \\
\text { comportamentais, como irritabilidade, } \\
\text { letargia/retraimento social e alterações } \\
\text { de sono e apetite. }\end{array}$ \\
\hline $\begin{array}{l}\text { Neece, } \\
\text { McIntyre, } \\
\text { Fenning. }\end{array}$ & $\begin{array}{l}\text { Estados } \\
\text { Unidos, } \\
2020\end{array}$ & $\begin{array}{l}\text { Examining the impact } \\
\text { of COVID-19 in } \\
\text { ethnically diverse } \\
\text { families with young } \\
\text { children with } \\
\text { intellectual and } \\
\text { developmental } \\
\text { disabilities }\end{array}$ & $\begin{array}{l}\text { Investigar as perspectivas dos } \\
\text { pais sobre o impacto do } \\
\text { COVID-19 em uma amostra } \\
\text { de famílias } \\
\text { predominantemente } \\
\text { hispânicas/latino-americanas } \\
\text { de crianças pequenas com } \\
\text { atraso de desenvolvimento ou } \\
\text { TEA que vivem nos EUA. }\end{array}$ & $\begin{array}{l}\text { Estudo } \\
\text { qualitativo }\end{array}$ & $\begin{array}{l}\text { Alguns pais também disseram que } \\
\text { seus filhos continuaram a ter ganhos } \\
\text { de desenvolvimento, principalmente } \\
\text { na linguagem, desde que estavam em } \\
\text { casa, apesar de experimentarem uma } \\
\text { redução nos serviços. }\end{array}$ \\
\hline $\begin{array}{l}\text { Nonweiler et } \\
\text { al. }\end{array}$ & \begin{tabular}{|l|} 
Reino \\
Unido, 2020
\end{tabular} & $\begin{array}{l}\text { Prevalence and } \\
\text { Associated Factors of } \\
\text { Emotional and } \\
\text { Behavioural } \\
\text { Difficulties during } \\
\text { COVID-19 Pandemic } \\
\text { in Children with } \\
\text { Neurodevelopmental } \\
\text { Disorders }\end{array}$ & $\begin{array}{l}\text { Examinar a prevalência de } \\
\text { problemas emocionais e } \\
\text { comportamentais e os fatores } \\
\text { associados a esses sintomas } \\
\text { em crianças com distúrbios } \\
\text { do neurodesenvolvimento } \\
\text { durante os estágios iniciais da } \\
\text { pandemia do COVID-19. }\end{array}$ & $\begin{array}{l}\text { Estudo } \\
\text { transversal }\end{array}$ & $\begin{array}{l}\text { Aqueles com TEA exibiram } \\
\text { comportamentos pró-sociais } \\
\text { diminuídos. Meninas com TEA } \\
\text { apresentaram sintomas emocionais } \\
\text { mais elevados em comparação com os } \\
\text { meninos. }\end{array}$ \\
\hline $\begin{array}{l}\text { O'Sullivan et } \\
\text { al. }\end{array}$ & $\begin{array}{l}\text { Irlanda, } \\
2021\end{array}$ & $\begin{array}{l}\text { A Qualitative Study of } \\
\text { Child and Adolescent } \\
\text { Mental Health } \\
\text { during the COVID-19 } \\
\text { Pandemic in Ireland }\end{array}$ & $\begin{array}{l}\text { Compreender as vivências de } \\
\text { crianças e adolescentes } \\
\text { durante o COVID-19. }\end{array}$ & $\begin{array}{l}\text { Estudo } \\
\text { qualitativo }\end{array}$ & $\begin{array}{l}\text { A mudança drástica na rotina } \\
\text { provocou ansiedade entre as crianças } \\
\text { com autismo. Os alunos com TEA } \\
\text { tinham uma compreensão abrangente } \\
\text { do vírus que provocou o medo de } \\
\text { COVID-19. Esta consciência de } \\
\text { COVID-19 provocou ansiedade } \\
\text { existencial não adaptativa } \\
\text { categorizada por um medo } \\
\text { avassalador do vírus. }\end{array}$ \\
\hline
\end{tabular}


Research, Society and Development, v. 10, n. 3, e57010313664, 2021

(CC BY 4.0) | ISSN 2525-3409 | DOI: http://dx.doi.org/10.33448/rsd-v10i3.13664

\begin{tabular}{|c|c|c|c|c|c|}
\hline Sergi et al. & Itália, 2021 & $\begin{array}{l}\text { Autism, Therapy and } \\
\text { COVID-19 }\end{array}$ & $\begin{array}{l}\text { Avaliar a estabilização e } \\
\text { generalização características } \\
\text { de uma intervenção baseada } \\
\text { em Análise Aplicada do } \\
\text { Comportamento (ABA) em } \\
\text { crianças afetadas com TEA } \\
\text { precoce. Investigaram a } \\
\text { manutenção de habilidades } \\
\text { previamente adquiridas e a } \\
\text { progressão de } \\
\text { comportamentos socialmente } \\
\text { significativos durante o } \\
\text { período de suspensão do } \\
\text { tratamento e durante os três } \\
\text { meses após o reinício do } \\
\text { tratamento pós-suspensão. }\end{array}$ & $\begin{array}{l}\text { Estudo } \\
\text { quantitativo }\end{array}$ & $\begin{array}{l}\text { Experimentaram melhorias na } \\
\text { comunicação, socialização e } \\
\text { autonomia pessoal durante o } \\
\text { lockdown. No mesmo período, o } \\
\text { comportamento de "compartilhar e } \\
\text { buscar o outro" aumentou e melhorou. } \\
\text { Aumento de estereótipos e } \\
\text { ritualização, uma nova mudança na } \\
\text { rotina diária ocasionou um aumento } \\
\text { nos comportamentos problemáticos. } \\
\text { Durante os três meses após o } \\
\text { tratamento ABA as habilidades } \\
\text { adquiridas foram mantidas, mas } \\
\text { nenhuma melhora significativa foi } \\
\text { demonstrada. }\end{array}$ \\
\hline $\begin{array}{l}\text { Siracusano et } \\
\text { al. }\end{array}$ & Itália, 2021 & $\begin{array}{l}\text { The Impact of COVID- } \\
19 \text { on the Adaptive } \\
\text { Functioning, } \\
\text { Behavioral Problems, } \\
\text { and Repetitive } \\
\text { Behaviors of Italian } \\
\text { Children with Autism } \\
\text { Spectrum Disorder: An } \\
\text { Observational Study }\end{array}$ & $\begin{array}{l}\text { Investigar o impacto do } \\
\text { lockdown COVID-19 no } \\
\text { funcionamento adaptativo, } \\
\text { problemas comportamentais e } \\
\text { comportamentos repetitivos } \\
\text { de crianças com TEA. }\end{array}$ & $\begin{array}{l}\text { Estudo } \\
\text { observacional }\end{array}$ & $\begin{array}{l}\text { Nenhuma piora significativa no } \\
\text { funcionamento adaptativo, } \\
\text { comportamentos problemáticos e } \\
\text { repetitivos surgiram após o } \\
\text { confinamento domiciliar obrigatório. } \\
\text { Nas crianças em idade escolar, a } \\
\text { estabilidade clínica foi encontrada em } \\
\text { referência às habilidades adaptativas e } \\
\text { aos aspectos comportamentais, ao } \\
\text { passo que, entre os pré-escolares, uma } \\
\text { melhoria significativa nas habilidades } \\
\text { adaptativas emergiu. }\end{array}$ \\
\hline $\begin{array}{l}\text { Türkoğlu et } \\
\text { al. }\end{array}$ & $\begin{array}{l}\text { Turquia, } \\
2020\end{array}$ & $\begin{array}{l}\text { The relationship } \\
\text { between chronotype, } \\
\text { sleep, and } \\
\text { autism symptom } \\
\text { severity in children } \\
\text { with ASD in } \\
\text { COVID-19 home } \\
\text { confinement period }\end{array}$ & $\begin{array}{l}\text { Investigar a relação entre } \\
\text { preferência de } \\
\text { cronótipo/problemas de sono } \\
\text { e gravidade dos sintomas de } \\
\text { crianças com transtorno do } \\
\text { espectro do autismo (TEA) } \\
\text { durante o confinamento e } \\
\text { isolamento social do surto de } \\
\text { COVID-19. }\end{array}$ & $\begin{array}{l}\text { Estudo } \\
\text { quantitativo }\end{array}$ & $\begin{array}{l}\text { Problemas de sono medeiam a relação } \\
\text { entre a pontuação do cronótipo e a } \\
\text { gravidade dos sintomas de autismo } \\
\text { sob a condição de confinamento } \\
\text { domiciliar COVID-19. }\end{array}$ \\
\hline
\end{tabular}

Fonte: Autores (2021).

\section{Considerações Finais}

A pandemia do COVID-19 gerou na vida de muitas pessoas uma série de mudanças, principalmente em suas rotinas. Para crianças com TEA, o distanciamento social causou problemas de ordem psicossocial, advindos, principalmente, da falta de interação social e da mudança abrupta da rotina. Sendo assim, para as crianças, essas mudanças são um processo de adaptação lento, visto que a manutenção da rotina é um ponto importante para os autistas, o que pode gerar ansiedade, estresse e medo. Apesar de a nova realidade ser assustadora, é importante tentar implementar uma rotina que remete àquela anteriormente vivenciada a pandemia. Além disso, é importante preparar as crianças de forma cautelosa e saudável, para que isso lhes traga calma ao invés de despertar comportamentos agressivos e ansiosos. Sendo assim, é necessário que as famílias com crianças portadoras de TEA busquem englobar um espaço dinâmico em suas casas para que esses indivíduos possam se comunicar e continuar seu 
desenvolvimento e possibilitar a adaptação à uma nova rotina, contribuindo para uma vivência equilibrada e saudável, visando o bem estar da criança.

Por fim, são necessários estudos a respeito de ferramentas lúdicas ou tecnológicas que auxiliem os pais/cuidadores de crianças com TEA a minimizarem os impactos negativos do isolamento social adotado durante a pandemia do COVID-19. Além de pesquisas sobre adoção de teleducação e telessaúde para manutenção do desenvolvimento infantil e da terapia ABA.

\section{Financiamento}

Para a condução desse estudo não houve financiamento por agências de fomento, sendo de inteira responsabilidade dos autores todos os custos oriundos para a construção deste manuscrito.

\section{Conflitos de Interesse}

Os autores declaram que não existem interesses concorrentes.

\section{Referências}

American Psychiatric Association.(2013). Diagnostic and Statistical Manual of Mental Disorers, (DSM-V).

Amorim, R, Catarino, S, Miragaia, P., Ferreras, C. Viana, V. \& Guardiano, M. (2020). Impacto de la COVID-19 en niños con trastorno del espectro autista. Rev. Neurol., 71(1), 285-291. https://neurologia.com/noticia/7898/impacto-de-la-covid-19-en-ninos-con-trastorno-del-espectro-autista.

Araujo, A. S. M. (2020). Estudo metodológico sobre o comportamento de crianças com transtorno do espectro autista (TEA) durante a pandemia da COVID-19. 26f. Trabalho de Conclusão de Curso (Graduação em Farmácia) - Departamento de Farmácia, Centro Ciências da Saúde, Universidade Federal do Rio Grande do Norte, Natal.

Autismo: em tempos de coronavírus como podemos ajudar? (2020).

Brito, A. R, Almeida, R.S, Crenze, L. G, Alves, A. S. M, Lima, R. C, \& Abranches, CD. (2020). Os desafios da pandemia da COVID-19 para autistas. Academia Brasileira de Neurologia. https://www.abneuro.org.br/post/os-desafios-da-pandemia-da-covid-19-para-autistas.

Barbosa, A, Figueiredo, A, Viegas, M, \& Batista, R. (2020). Os impactos da pandemia covid-19 na vida das pessoas com transtorno do espectro autista. Revista Da Seção Judiciária Do Rio De Janeiro, 24(48), 91-105.

Colizzi, M., Sironi, E., Antonini, F., Ciceri, M. L., Bovo, C., \& Zoccante. L. (2020). Psychosocial and Behavioral Impact of COVID-19 in Autism Spectrum Disorder: An Online Parent Survey. Brain Sciences. 10(6), p. 341. https://www.mdpi.com/2076-3425/10/6/341.

Costa, D. D. S, Loureiro, F, Moreira, L, Silveira, B. K. S, Sadi, H. D. M., Apolinário-Souza, Tércio, \& Pinheiro, M. I. C. (2020). Saúde Mental na pandemia de Covid-19: considerações práticas multidisciplinares sobre cognição, emoção e comportamento. Debates em psiquiatria - Ahead of print. https://www.researchgate.net/publication/341255949_Saude_mental_na_pandemia_de_COVID_-19_consideracoes_praticas_multidiscipli nares_sobre_cognicao_emocao_e_comportamento.

Baumer, N, \& Spence, S. J. (2018). Evaluation and Management of the Child With Autism Spectrum Disorder. continuum: Lifelong Learning in Neurology, 24, 248-275. https://pubmed.ncbi.nlm.nih.gov/29432246/.

Colizzi, M., Sironi, E., Antonini, F., Ciceri, M. L., Bovo, C., \& Zoccante, L. (2020). Psychosocial and Behavioral Impact of COVID-19 in Autism Spectrum Disorder: An Online Parent Survey. Brain Sciences. 10(6), 341. https://pediatrics.aappublications.org/content/145/1/e20193447.

Deslandes, S. F, \& Coutinho, T. (2020). O uso intensivo da internet por crianças e adolescentes no contexto da COVID-19 e os riscos para violências autoinflingidas. Ciência \& Saúde Coletiva. 25(1), 2479-2486, 2020. https://www.scielo.br/scielo.php?script=sci_arttext\&pid=S1413$81232020006702479 \& \operatorname{tlng}=$ pt.

Farias, M. N. \& Leite Júnior, J. D. (2020) Vulnerabilidade social e Covid-19: considerações a partir da terapia ocupacional social. Cadernos Brasileiros de Terapia Ocupacional.

Fernandes, A. D. S. A., Speranza, M, Mazak, M. S. R., Gasparini, D. A., \& CID, M. F. B. (2020). Desafios cotidianos e possibilidades de cuidado às crianças e adolescentes com Transtorno do Espectro Autista (TEA) frente à COVID-19. Cad. Brasileiros de Terapia Ocupacional. 
Research, Society and Development, v. 10, n. 3, e57010313664, 2021 (CC BY 4.0) | ISSN 2525-3409 | DOI: http://dx.doi.org/10.33448/rsd-v10i3.13664

Houting, J. (2020). Stepping out of isolatin: autistic people and Covid-19. Autism in Adulthood. 2(2), 1-3. https://www.liebertpub.com/doi/10.1089/ AUT.2020.29012.JDH

Jeste, S, Hyde, C, Distefano, C, Halladay, A, Ray, S, Porath, M, Wilson, R. B, \& Thurm, A. (2020). Changes in access to educational and healthcare services for individuals with intellectual and developmental disabilities during COVID-19 restrictions. Journal of Intellectual Disability Research, 64, 825-833. https://onlinelibrary.wiley.com/doi/full/10.1111/jir.12776.

Kentaro, K., Rie, H., Kiwamu, N., Ayumi, Y., Fumie, H., \& Shu-Ichi, U. (2020) Excessive and Problematic Internet Use During the Coronavirus Disease 2019 School Closure: Comparison Between Japanese Youth With and Without Autism Spectrum Disorder. Frontiers in Public Health, 17(8), 609347, https://pubmed.ncbi.nlm.nih.gov/33392143/.

Mendes, K. D. S, Silveira, R. C. C. P, \& Galvão, C. M. (2008). Revisão integrativa: método de pesquisa para a incorporação de evidências na saúde e na enfermagem. Texto contexto - enferm, 17(4), 758-764. https://www.scielo.br/pdf/tce/v17n4/18.pdf.

Moher, D, Liberati, A, Tetzlaff, J, \& Altman, D. G. (2009). Preferred Reporting Items for Systematic Reviews and Meta-Analyses: The PRISMA Statement. PLoS Med, 6(7), p.e1000097. https://journals.plos.org/plosmedicine/article?id=10.1371/journal.pmed.1000097.

Mumbardó-Adam, C, Barnet-López, S, \& Balboni, G. (2021). How have youth with Autism Spectrum Disorder managed quarantine derived from COVID-19 pandemic? An approach to families perspectives. Research in Developmental Disabilities, 110. https://pubmed.ncbi.nlm.nih.gov/334863 95.

Neece, C, Mcintyre, L. L, Fenning, R. (2020). Examining the impact of COVID-19 in ethnically diverse families with young children with intellectual and developmental disabilities. Journal of Intellectual Disability Research, 64(10), 739- 749. https://pubmed.ncbi.nlm.nih.gov/32808424/.

Nonweiler, J, Rattray, F, Baulcomb, J, Happé, F, \& Absoud, M. (2020). Prevalence and Associated Factors of Emotional and Behavioural Difficulties during COVID-19 Pandemic in Children with Neurodevelopmental Disorders. Children, 7(9),128. https://pubmed.ncbi.nlm.n ih.gov/328997 99/.

Organização Pan-Americana de Saúde - OPAS. (2020) Organização Mundial da Saúde - OMS. Folha informativa - Covid-19 (doença causada pelo novo coronavírus).

Organización Mundial de la Salud - OMS. (2020). Brote de enfermedad por coronavirus (COVID-19).

O’Sullivan, K, Clark, S, Mcgrane, A, Rock, N, Burke, L, Boyle, N, Joksimovic, N, \& Marshall, K. (2021) A Qualitative Study of Child and Adolescent Mental Health during the COVID-19 Pandemic in Ireland. International Journal of Environmental Research and Public Health, 18(3).

Souza, M. T, Silva, M. D, \& Carvalho, R. (2010). Revisão integrativa: o que é e como fazer Einstein, 8(1), 102-106. https://www.scielo.br/pdf /eins/v8n1/pt_1679-4508-eins-8-1-0102.pdf.

Transtorno do Espectro Autista. (2019). Sanar Med.

Sergi, L., Mingione, E., Ricci, M. C, Cavallaro, A, Russo, F, Corrivetti, G, Operto, F. F, \& Frolli, A. (2021) Autismo, terapia e COVID-19. Pediatr. Rep., 13(1), 35-44. https://ww .sbp.com.br/fileadmin/user_upload/22455c-NA_-_COVID-19_e_Transtorno_do_Espectro_Autista_1_.pdf.

Siracusano, M, Segatori, E, Riccioni, A, Gialloreti, L. E, Curatolo, P, \& Mazzone, L. (2021). The Impact of COVID-19 on the Adaptive Functioning, Behavioral Problems, and Repetitive Behaviors of Italian Children with Autism Spectrum Disorder: An Observational Study. Children, 8( 2). https://pubmed.ncbi.nlm.nih.gov/33540683/

Tuba, M, Ceymi, D, \& Herdem, A. G. (2020). Behavioral Implications of the Covid-19 Process for Autism Spectrum Disorder, and Individuals Comprehension of and Reactions to the Pandemic Conditions. Frontiers in Psychiatry, 11, https://www.frontiersin.org/articles/10.3389/fpsyt.2020.5 6188 2/full.

Türkoğlu, S, Uçar, N. H, Çetin, F. H, Güler, H. Á, \& Tezcan, M. E. (2021) The relationship between chronotype, sleep, and autism symptom severity in children with ASD in COVID-19 home confinement period. Chronobiology International, 37(8), 12071213, https://pubmed.ncbi.nlm.nih.gov/32746638/.

World Health Organization. (2020). WHO Director-General's opening remarks at the media briefing on COVID19. 